\title{
DISEÑO DE UNA MÁQUINA COSECHADORA DE ZANAHORIA ${ }^{31}$
}

Lazo Baltazar Brecio Daniel ${ }^{1}$, Huatuco Gonzales Mario²

Facultad de Ingeniería Mecánica de la Universidad Nacional del Centro del Perú

\begin{abstract}
RESUMEN
La investigación se ha enfocado en el estudio del diseño de una máquina encargada para la cosecha de la zanahoria en el Valle del Mantaro. Para realizar el correspondiente diseño de la máquina se tuvo que visitar en 5 oportunidades al lugar de dicha actividad, donde se tomaron anotaciones como son características y parámetros que se tiene en cuenta del momento de la cosecha, también se conversó con el respectivo dueño para obtener una idea clara sobre características de la futura máquina, lo mencionado se plasmó en la lista de exigencias. Luego se desarrolló una estructura de funciones acorde a las actividades desarrolladas en la cosecha de la zanahoria, donde se clasificó y diferenció los procesos que va desarrollar la máquina. Mediante una matriz morfológica y la combinación en esta, se planteó tres conceptos de los cuales se eligió el más óptimo mediante una evaluación técnica-económica. Por último, se empezó a dimensionar y seleccionar el tipo de material de las partes de la máquina, para lo cual se tuvo en cuenta dimensiones básicas como son; dimensiones de los tablones, dimensiones requeridas según la lista de exigencia.
\end{abstract}

Palabras Clave: Diseño, máquina cosechadora, zanahoria

\section{DESIGN OF A CARROT HARVESTER MACHINE}

\section{ABSTRACT}

The investigation has focused on the study design of a machine responsible for the harvest of carrots in the Mantaro Valley. To make the design for the machine had 5 opportunities to visit on the site of this activity, where notes like characteristics and parameters were taken into account at the time of harvest It was also talked to the respective owner to obtain a clear idea about the future characteristics of the machine, the above is reflected in the list of requirements. Then it was developed a structure of functions according to the activities developed in the carrot crop, which classify and differentiate the processes that will develop the machine. Using a morphological matrix and the combination in this, we proposed three concepts from which was chosen the most optimal through a technical and economic evaluation. Finally it began to size and select the type of material parts of the machine, which took into account basic dimension as they are, size of the boards, dimensions required by the list of requirements.

Key words: Design, Machine, harvester, carrot.

31 Trabajo de investigación fue recibido el 07/05/2010 retornado para su revisión el 15/06/2010 y aprobado para su publicación 16/11/2010 


\section{INTRODUCCIÓN}

La zanahoria es una hortaliza por excelencia que tiene múltiples ventajas en la alimentación de las personas de todas las edades. Es un recurso terapéutico más valioso para tratar los padecimientos de pérdida de la vista por concentrar en sus raíces minerales y vitaminas.

La forma de sembrado en el Valle del Mantaro es del modo de voleo (como la cebada) en surcos y tablones, lo cual provoca que en la cosecha se presenten algunos inconvenientes, ya que la zanahoria no está distribuida de forma ordenada como en el caso de la papa (surcos).

En el Valle del Mantaro la producción de zanahoria es durante todo el año y la temperatura tolerante antes de la cosecha para una buena calidad del producto debe de ser de $15-18^{\circ} \mathrm{C}$.

El inconveniente que se tiene hasta el momento a lo que se refiere a este hortaliza, es en la cosecha, ya que hasta el momento se está realizando en forma artesanal (manual), debido a que las hojas de la zanahoria no son tan fuertes para soportar el jalón de la tierra, por lo que cavar ayuda a quitar la zanahoria sin algún daño para lo cual se utiliza: picota o pico de uña y arrancando la planta entera con sus hojas, las cuales se cortan después al ras del cuello. Las raíces se lavan con agua abundante para retirar la tierra adherida.

En este contexto, el presente trabajo de investigación titulado Diseño de una máquina cosechadora de zanahoria pretende solucionar el problema de la cosecha de la zanahoria, para realizar el diseño se utilizó la hipótesis siguiente: Si diseñamos una maquina utilizando la tecnología apropiada, entonces adecuaremos a la cosecha de zanahorias en el Valle del Mantaro

Entonces la tarea para solucionar el problema fue, diseñar una maquina adecuada para realizar la cosecha de zanahorias en el Valle del Mantaro.

\section{MATERIALES Y MÉTODOS}

\section{Material}

La tarea técnica que presenta la organización planificadora o el cliente y que determinan los parámetros de las máquinas, la esfera y las condiciones de su empleo.

La proposición técnica planteada en orden de iniciativa por la oficina de proyectos o un grupo de diseñadores.

El trabajo de investigación científica o el prototipo experimental creado por el investigador.
La sugerencia de invención o el modelo experimental creado por el investigador.

El modelo de la máquina que se debe copiar o reproducir con modificaciones.

En este caso la tarea técnica lo presenta el cliente, el cual determina los parámetros de la máquina y las condiciones de su empleo.

\section{Métodos}

Se ha utilizado la metodología de diseño según la norma VDI 2221/2222. Esta metodología incluye los pasos de todas las metodologías estudiadas y agrupa los pasos en cuatro partes principales:

\section{Planteamiento del problema.}

Tarea, aclaración y precisión de la tarea.

\section{Concepción de la solución.}

Definición de funciones y estructura, búsqueda de los principios de solución.

\section{Elaboración del proyecto.}

División de módulos realizables, configuración dimensional de módulos, configuración del producto.

\section{Elaboración de detalles.}

Elaboración de detalles para la ejecución y aprovechamiento, solución constructiva.

Para desarrollar el trabajo de investigación se ha utilizado el método de abstracción.

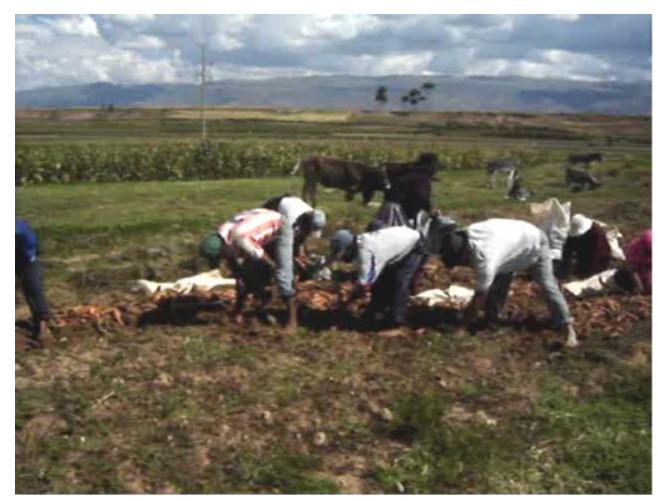




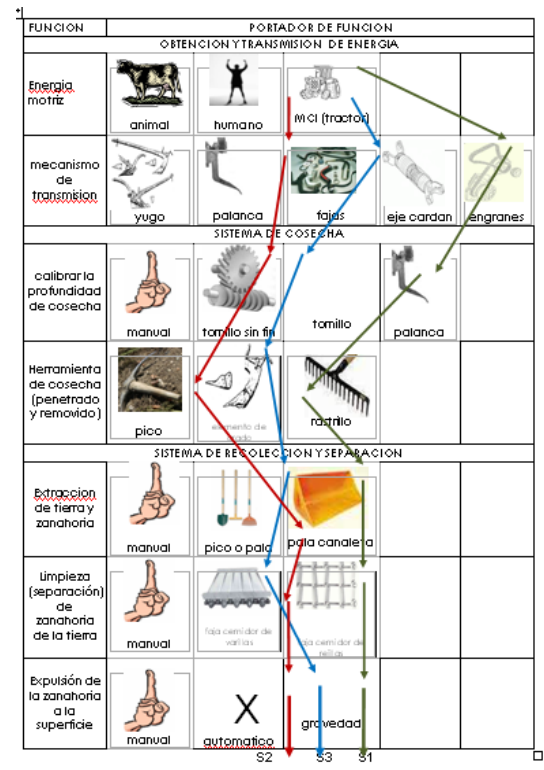

Fig. 1.1 Cosecha de la Zanahoria

Dentro de la caja negra (Black box) se supone que ocurre un proceso técnico, es decir una transformación de las propiedades de las tres magnitudes básicas.

Materia: Materia prima, insumos, productos terminados, piezas, fluidos, granulados, objetos de todo tipo.

Energía: Energía mecánica, térmica, eléctrica, química, óptica, energía atómica, etc.

Señal: Magnitudes de medición, datos, valores indicados, impulsos de control, información.

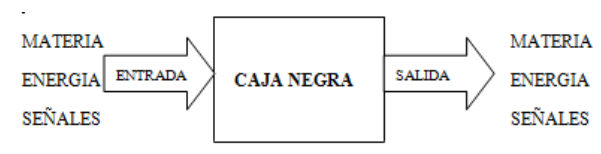

\begin{tabular}{|c|c|c|}
\hline & ENTRADA & SALIDA \\
\hline MATERIA & SEMBRIO DE ZANAHORIA & ZANAHORIA COSECHADA \\
\hline ENERGIA & 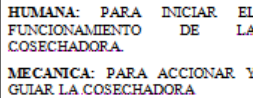 & RUIDO, VIBRACIONES, CALOR \\
\hline SEÑALES & $\begin{array}{llll}\text { ACTIVADA AL DAR } & \text { INCIO } & \text { AL } \\
\text { FUNCIONAMIENTO } & \text { DE } & \text { LA } \\
\text { MAQUNA } & & & \\
\end{array}$ & $\begin{array}{l}\text { QUE INDIQUE EL DESPLAZANIENTO DE } \\
\text { LA.COSECHADORA.Y SU PARADA }\end{array}$ \\
\hline
\end{tabular}

Fig. 1.2 Caja negra

\section{RESULTADOS}

Utilizando el marco teórico de la abstracción se llega a definir el concepto óptimo de la máquina.

\section{Determinación del concepto óptimo.}

Se trata de la búsqueda de las soluciones. Lo que aquí se presenta es una de las formas de llegar a las soluciones con la ayuda de un esquema ordenado: La matriz morfológica.

\section{Cuadro 1.1 : Matriz morfológica}

Fig. 1.3 Concepto de solución 1.
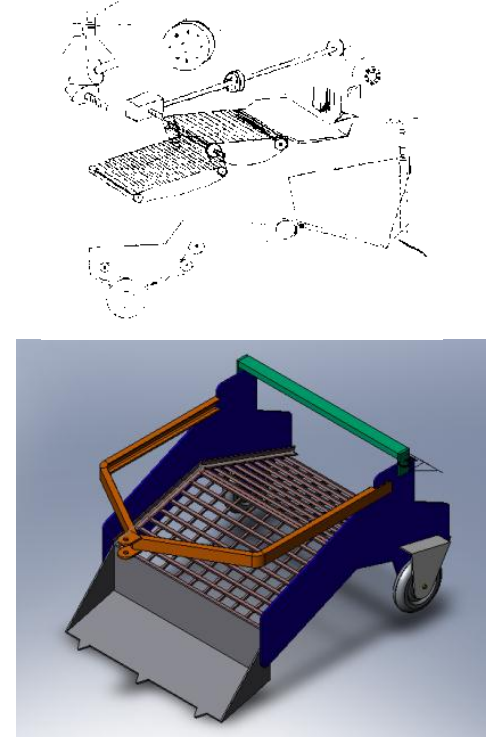

Fig.1.4 Concepto de solución 2.

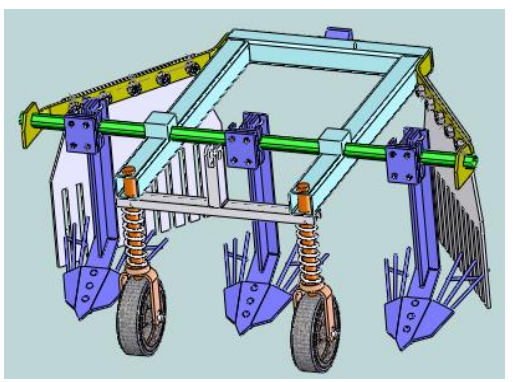

Fig.1.5 Concepto de solución 3.

DISCUSIÓN

Mediante una evaluación técnica económica logramos elegir la máquina óptima.

Tabla 1.1 Evaluación técnica y el factor de importancia. 


\begin{tabular}{|c|c|c|c|c|c|c|}
\cline { 2 - 6 } \multicolumn{1}{c|}{} & \multirow{2}{*}{$\begin{array}{c}\text { Puntos de } \\
\text { evaluación }\end{array}$} & \multirow{2}{*}{$\begin{array}{c}\text { Fac. D } \\
\text { import. }\end{array}$} & \multicolumn{5}{|c|}{ Proyectos } \\
\cline { 3 - 7 } 1 & Función & 3 & 2 & 2 & 2 & 4 \\
2 & Forma & 2 & 3 & 3 & 3 & 4 \\
3 & Diseño & 2 & 1 & 2 & 3 & 4 \\
4 & Seguridad & 3 & 2 & 3 & 2 & 4 \\
5 & Ergonomía & 2 & 1 & 2 & 3 & 4 \\
6 & Fabricación & 2 & 1 & 4 & 4 & 4 \\
7 & Montaje & 1 & 1 & 3 & 3 & 4 \\
8 & Uso & 1 & 2 & 1 & 4 & 4 \\
9 & Mantenimiento & 1 & 2 & 3 & 3 & 4 \\
\hline \multicolumn{7}{|c|}{ Total= } \\
\cline { 3 - 7 } & Coef. Técnico= puntj & 29 & 44 & 48 & 68 \\
\hline total/puntj ideal & 0.4265 & 0.6471 & 0.7059 & 1 \\
\hline
\end{tabular}

Tabla 1.2 Evaluación económica y el factor de importancia.

\begin{tabular}{|c|c|c|c|c|c|c|}
\hline \multirow{2}{*}{\multicolumn{2}{|c|}{$\begin{array}{l}\text { Puntos de } \\
\text { evaluación }\end{array}$}} & \multirow{2}{*}{\begin{tabular}{|l|} 
Fact. \\
Impor.
\end{tabular}} & \multicolumn{4}{|c|}{ Proyectos } \\
\hline & & & \multirow{2}{*}{$\frac{\text { A. } 1}{2}$} & \multirow{2}{*}{ A.2 } & \multirow{2}{*}{$\frac{\text { A.3 }}{4}$} & \multirow{2}{*}{\begin{tabular}{|l|} 
A.4 \\
4
\end{tabular}} \\
\hline 1 & $\begin{array}{l}\text { Costo de } \\
\text { material }\end{array}$ & 3 & & & & \\
\hline 2 & Costo de & 3 & 1 & 3 & 3 & 4 \\
\hline 3 & $\begin{array}{l}\text { Costo de } \\
\text { mano de } \\
\text { obra }\end{array}$ & 3 & 1 & 3 & 3 & 4 \\
\hline \multicolumn{3}{|c|}{ Total $=$} & 12 & 30 & 30 & 36 \\
\hline \multicolumn{3}{|c|}{$\begin{array}{l}\text { Coefi. Econ. = puntj. } \\
\text { Total/puntj. Ideal }\end{array}$} & 0.333 & 0.833 & 0.833 & 1.000 \\
\hline
\end{tabular}

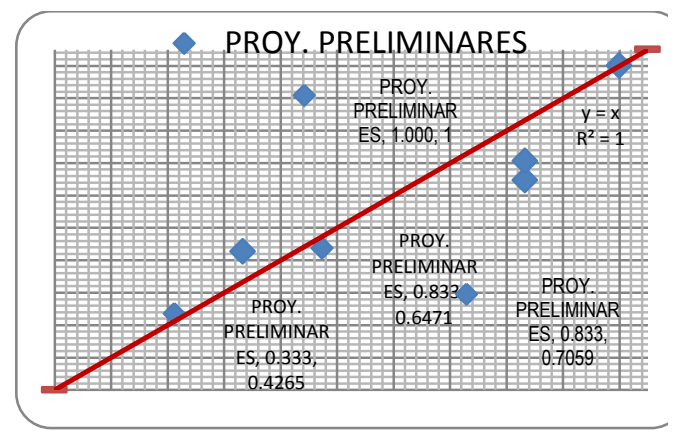

Fig.1.6 Diagrama economico vs tecnico

Podemos observar en el fig. 1.6 Diagrama económico vs técnico, que la solución tres es la mejor alternativa.

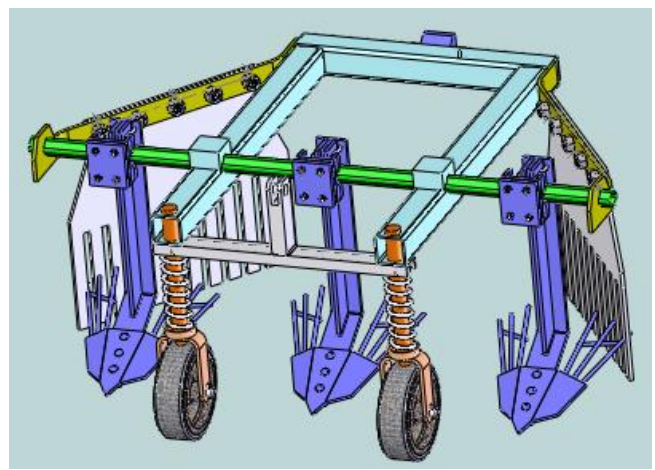

Fig. 1.7 Cosechadora de zanahoria.

\section{CONCLUSIONES}

- La técnica de revisión bibliográfica los deseos y exigencias de los agricultores fueron parte importante para obtener el concepto óptimo de la cosechadora.

- Los modelos, y el test de experimentación realizados al entorno de los productores de la zanahoria dieron lugar a ubicar la mejor máquina.

- Con las pruebas realizadas en un simulador se realizaron los ajustes necesarios para luego elaborar los planos de fabricación de la cosechadora. La máquina diseñada cumple con las exigencias establecida por los productores de zanahoria.

- El tamaño de la máquina se estipuló según medidas básicas como son ancho de los tablones y profundidad de penetración.

- El diseño de la máquina tiene un costo al alcance de los agricultores.

- Los materiales que se utilizarán para la fabricación, se encuentra en el mercado local, como son las representantes de las marcas Sider Perú y Aceros Arequipa.

- Utilizando el concepto de sostenibilidad y el marco teórico de Eco diseño se logra disminuir el impacto de la contaminación del medio ambiente debido a los residuos ocasionados por los procesos tecnológicos de los cambios cualitativos.

- El mantenimiento de la máquina será fácil, debido a que no cuenta con numerosas partes móviles y no necesita de lubricantes.

\section{REFERENCIAS BIBLIOGRÁFICAS}

- Barriga Benjamín, 1985, "Curso de Diseño Mecánico 1", PUCP, Lima Perú. 
- Roth K., 1994, "Konstruieren Mit Konstruktionskatalogen", Springer - Verlag, BerlinHeidelberg.

- Niemann Gustav, 1994, "Elementos de máquinas", Vol. 1, Labor, Barcelona.

- $\quad$ Norton Robert L., 1996, "Machine Design And Integrated Approach", Prentice Hall, Upper Saddle River, New Jersy.

- Pahl G. and Beitz W., 1996, "Engineering Design", Springer-Verlag, Berlin-Heidelberg.

- V. Korsakov, 1985, "Fundamentos de la Tecnología en la Construcción De Maquinaria", Editorial MIR Moscú.

- G. G. Baranov, 1985, "Teórica de los Mecanismos y Maquinas", Editorial MIR Moscú. 